\title{
The diagnostic role of Saliva - A Review.
}

\author{
Sanjeev Mittal ${ }^{1}$, Vikram Bansal ${ }^{2}$, Sushant Garg ${ }^{3}$, Gaurav Atreja ${ }^{4}$, Sanjay Bansal ${ }^{1}$ \\ ${ }^{1}$ Professor, Dept of Prosthodontics, M.M.College of Dental Sciences \& Research, Mullana, Ambala, Haryana. \\ ${ }^{2}$ Lecturer, Dept of Public Health Dentistry, M.M.College of Dental Sciences \& Research, Mullana Ambala, Haryana. \\ ${ }^{3}$ Professor \& HOD, Dept of Prosthodontics, M.M.College of Dental Sciences \& Research, Mullana, Ambala, Haryana. \\ ${ }^{4}$ Lecturer, Dept of Prosthodontics, M.M.College of Dental Sciences \& Research, Mullana, Ambala, Haryana.
}

Correspondence:

Dept of Public Health Dentistry

M.M.College of Dental Sciences \& Research,

Mullana (Ambala)

Haryana, India.

Email: vikrambansal2001@gmail.com

\begin{abstract}
As a diagnostic fluid, saliva offers distinctive advantages over serum because it can be collected non-invasively by individuals, even by patient. Does not require special equipment for collection and storage as unlike blood saliva does not clot. Advantageous for person in whom blood drawing is difficult as in obese and haemophilic patient. Whole saliva used for diagnosis of systemic diseases, because it contains serum constituents. These constituents are derived from the local vasculature of the salivary glands and gingival cervicular fluid.This review examines the diagnostic application of saliva for hereditary disorders, autoimmune diseases, malignant and infectious diseases, and endocrine disorders, as well as in the assessment of therapeutic levels of drugs and the monitoring of illicit drug use, and also for forensic evidence and others. . In future we are likely to see the increased utilization of saliva as a diagnostic fluid. As we enter the era of genomic medicine, sialochemistry will play an increasingly important role in the early detection, the monitoring and progression of the systemic and oral diseases.
\end{abstract}

Key words: saliva, diagnosis, diseases, disorders, alcohol, caries 


\section{Introduction}

Most commonly used laboratory diagnostic procedures involve the analyses of the cellular and chemical constituents of blood. Saliva offers some distinctive advantages when used for diagnosis of disease. Whole saliva can be collected non-invasively, and by individuals with limited training, including the patient. No special equipment is needed for collection of the fluid. Valuable for children, since collection of the fluid is associated with fewer compliance problems. Further, analysis of saliva may provide a cost-effective approach for the screening of large populations. Advances in the use of saliva as a diagnostic fluid have been affected by current technological developments: enzyme-linked fluorescence technique, Western blot assays, polymerase chain reaction (PCR).

Whole saliva is a mixed fluid that is derived predominantly from major and minor salivary glands. Whole saliva also contains gingival cervicular fluid, mucosal transdute, expectorated bronchial and nasal secretions. Serum and blood derivatives from oral wound, bacteria, bacterial products, viruses and fungi, desquamated epithelial cells, other cellular component, and food debris. Saliva is approximately $99 \%$ water and $1 \%$ protein and salts. Normal daily salivary flow range from 0.5 to $1.5 \mathrm{~L}$ with average volume of $1 \mathrm{ml}$ at any given time.

Saliva can be considered as gland-specific saliva and whole saliva. Evaluation of the secretions from the individual salivary glands are primarily useful for the detection of gland-specific pathology, i.e., infection and obstruction. However, whole saliva is most frequently studied when salivary analysis is used for the evaluation of systemic disorders.

Principal functions of saliva are lubrication to protect the oral mucosa. It also aids in the digestion of food, cleansing of oral cavity, also facilitate speech, neutralizing unwanted effect of acid on oral tissue by buffering action, maintenance of supersaturated calcium phosphate concentrations and antimicrobial action.

Analysis of saliva done for the diagnosis of following -

1. Hereditary disease

2. Autoimmune disease

3. Malignancy

4. Infection

5. Monitoring of levels of hormones

6. Monitoring of levels of drugs.

7. Bone turnover marker in saliva

8. Forensic Evidence

9. Dental caries and periodontal disease

10. Diagnosis of Oral Disease with Relevance for Systemic Diseases

1. Hereditary diseases

Cystic fibrosis $(C F)$ is a genetically transmitted disease of children and young adults, which is considered a generalized exocrinopathy. A defective electrolyte trans- port in epithelial cells and viscous mucus secretions from glands and epithelia characterize this disorder. The organs mostly affected in CF are: sweat glands, the lungs and the pancreas.

Elevations in electrolytes (sodium, chloride, calcium, and phosphorus), urea and uric acid, total protein and lipid were observed in the submandibuar saliva of CF patients (1). Most of the studies concerning the diagnostic application of saliva for CF are relatively old, and saliva is not currently used for the diagnosis of this disorder. 21-Hydroxylase deficiency is an inherited disorder of steroidogenesis which leads to congenital adrenal hyperplasia. Early morning salivary levels of 17-hydroxyprogesterone (17-OHP) determined by ELISA is an excellent screening test for the diagnosis of non-classic 21-hydroxylase deficiency, since the salivary levels accurately reflected serum levels of 17-OHP (2).

\section{Autoimmune diseases}

Sjögren's syndrome - Sjögren's syndrome (SS) is an autoimmune exocrinopathy of unknown etiology. Serum chemistry can demonstrate polyclonal hypergammaglobulinemia and elevated levels of rheumatoid factor, antinuclear antibody, anti-SS-A, and anti-SS-B antibody. In addition, increased concentrations of sodium and chloride, $\operatorname{IgA}$, IgG, lacoferrin, and albumin, and a decreased concentration of phosphate were reported in saliva of patients with SS (3).

No single salivary or serum constituent can accurately serve as a diagnostic marker for SS, the most important aspect of salivary diagnosis for this disease is the reduced salivary flow, although not pathognomonic for SS, is of clinical importance and can lead to a variety of oral signs and symptoms, such as progressive dental caries, fungal infections, oral pain, and dysphagia. Dentists are normally the first to encounter these patients. Affected individuals should be referred for a comprehensive evaluation of the cause for the reduced salivary flow.

\section{Malignancy}

Salivary analysis may aid in the early detection and screening of certain malignant tumors. Saliva also aids in monitoring the efficacy of treatment. The mRNA levels for specific proteins are elevated in the saliva of head and neck cancer patients.

p53 p53 is a tumor suppressor protein which is produced in cells exposed to various types of DNA-damaging stress. Inactivation of this suppressor through mutation is considered a frequent occurrence in the development of human cancer. Accumulation of inactive $\mathrm{p} 53$ protein occur, which in turn lead to the production of antibodies directed against this $\mathrm{p} 53$ protein. The $\mathrm{p} 53$ antibodies can be detected in the saliva of patients diagnosed with oral squamous cell carcinoma (SCC), and can thus assist in the early detection and screening for, this tumor (4).

Defensins Elevated levels of salivary defensin-1 were found to be indicative of the presence of oral SCC. A 
high-positive correlation was observed between salivary defensin-1 levels and serum levels of SCC-related antigen (5).

$C A 15-3$, c-erbB-2 Elevated levels of recognized tumor markers c-erbB-2 (erb) and cancer antigen 15-3 (CA15-3) were found in the saliva of women diagnosed with breast cancer, as compared with patients with benign lesions and healthy controls. They appear to hold greater promise for the early screening and detection of breast cancer (6).

CA 125 is a tumor marker for cancer. Elevated salivary levels of CA 125 were detected in patients with untreated breast cancer than healthy controls and patients who were treated for breast cancer. A positive correlation was found between salivary and serum levels of CA 125 (7). Higher levels of salivary nitrate and nitrite, and increased activity of nitrate reductase, were found in oral cancer patients compared with healthy individuals (8).

\section{Infectious diseases}

Saliva contains immunoglobulins (IgA, $\operatorname{IgM}, \operatorname{IgG}$ ) that originate from two sources: the salivary glands and serum. Antibodies against viruses, bacteria, fungal and parasite can be detected in saliva and can aid in the diagnosis of infections.

Helicobacter pylori infection has been associated with peptic ulcer and chronic gastritis. Oral cavity may be the source of infection. There was considerable variation in the detection rate of H.pylori DNA in salivary samples. There is controversy whether saliva act as permanent reservoir for this bacterium and gastric and salivary specimen harbor identical or different strains. PCR cannot distinguish between living and dead organism (9).

Shigella In the children infected with Shigella revealed higher titers of anti-Shiga toxin antibody in comparison with healthy controls (10).

Pneumococcal pneumonia the detection of pneumococcal $\mathrm{C}$ polysaccharide in saliva by ELISA may offer a valuable complement to conventional diagnostic methods for pneumococcal pneumonia. Quantitative measurement of pneumococcal capsular antigen in the saliva may be valuable in helping to make an aetiological diagnosis in children with pneumonia (11).

Lyme disease is caused by the spirochete Borrelia burgdorferi and is transmitted to humans by blood-feeding ticks. The detection of anti-tick antibody in saliva serves as a screening mechanism for individuals at risk for Lyme disease (12).

Taenia solium Specific antibody to Taenia solium larvae in serum demonstrated greater sensitivity than antibody in saliva for identification of neurocysticercosis. However, considering the simple and non-invasive nature of saliva sampling, it was suggested that saliva could be used in epidemiologic studies of this disease (13). Non invasive diagnosis of amebic liver abscess is challenging, detection of E. histolytica DNA in saliva by real- time PCR assay could be used as a diagnostic tool for amebic liver abscess (14).

Viral Diseases

HIV Antibody to HIV in whole saliva of infected individuals was detected by ELISA and Western blot assay, correlated with serum antibody levels (15). Salivary IgA levels to HIV decline as infected patients become symptomatic. It was suggested that detection of IgA antibody to HIV in saliva may, therefore, be a prognostic indicator for the progression of HIV infection. Analysis of antibody in saliva as a diagnostic test for HIV (or other infections) offers several distinctive advantages when compared with serum.

1. Saliva can be collected non-invasively, which eliminates the risk of infection for the health care worker who collects the blood sample.

2. Furthermore, viral transmission via saliva is unlikely, since infectious virus is rarely isolated from saliva.

3. Saliva collection also simplifies the diagnostic process in special populations in whom blood drawing is difficult, i.e., individuals with compromised venous access (e.g., injecting drug users), patients with hemophilia, and children

Several salivary and oral fluid tests have been developed for HIV diagnosis. Orasure is the only FDA- approved, commercially available testing system. It detects antibodies against the p24 antigen of HIV. The applicator swab is gently rubbed along the outer gums and inserted into a vial containing the developer solution that detects the antibody to p24 antigen of HIV (16). In conclusion, collection and analysis of saliva offer a simple, safe, well-tolerated, and accurate method for the diagnosis of HIV infection. Applicable for both clinical use and epidemiological surveillance

Hepatitis Saliva was found to be a useful alternative to serum for the diagnosis of viral hepatitis. Acute hepatitis A (HAV) and hepatitis B (HBV) were diagnosed based on the presence of IgM antibodies in saliva. Hepatitis B virus DNA revealed by PCR in saliva. Quantitative detection of DNA used to evaluate level of virus in the body copy for judgement of infection. It also point to the possible role of saliva as a source of HBV infection. $(17,18)$. Saliva has also been used for screening for hepatitis B surface antigen (HbsAg) in epidemiological studies.

Measles, mumps, and rubella Saliva may also be used for determining immunization and detecting infection with measles, mumps, and rubella (19).

Rotavirus For newborn infants, the salivary IgA response was found to be a better marker of rotavirus (RV) infection than the serum antibody response. Neonatal RV infection elicited specific mucosal antibody response which persisted for at least 3 months. However, a similar systemic immune response could not be observed, possibly due to interference by maternal antibody. Salivary 
antibodies could be used to monitor the immune response to vaccination and infection with RV (20).

Reactivation of herpes simplex virus type-1 (HSV-1) is involved in the pathogenesis of Bell's palsy and PCRbased identification of virus DNA in saliva is a useful method for the early detection of HSV-1 reactivation in patients with Bell's palsy (21).

Dengue is a mosquito-transmitted viral disease. Salivary levels of anti-dengue IgM and IgG demonstrated sensitivity of $92 \%$ and specificity of $100 \%$ in the diagnosis of infection. So, detection of dengue specific salivary $\operatorname{IgG}$ and $\operatorname{IgM}$ antibodies is useful markers for dengue infection (22).

\section{Detection of drugs}

Saliva has been proposed for the monitoring of systemic levels of drugs (Table 1). A fundamental prerequisite for this diagnostic application of saliva is a definable relationship between the concentration of a therapeutic drug in blood and the concentration in saliva. Only the unbound fraction of the drug in serum is available for diffusion into saliva the unbound fraction of a drug is usually the pharmacologically active fraction. This may represent an advantage of drug monitoring in saliva in comparison with drug monitoring in serum, where both bound and unbound fractions of a drug can be detected. Saliva may be used for monitoring patient compliance with psychiatric medications. Saliva is also useful for the monitoring of anti-epileptic drugs and anti-cancer drugs. Estimation of salivary carbamazepine levels is predictable and convenient method of drug monitoring in epileptic patient, and a positive correlation $(\mathrm{r}=0.659)$ between salivary and serum carbamazepine levels was observed (23).

\begin{tabular}{|l|l|}
\hline \multicolumn{2}{|l|}{ DRUG MONITORING IN SALIVA } \\
\hline Antipyrine & Phencyclidin \\
\hline Carbamazepine & Paracetamol \\
\hline Caffeine & Phenytoin \\
\hline Cisplatin & Primidone \\
\hline Cyclosporine & Quinine \\
\hline Diazepam & Procainamide \\
\hline Digoxin & Sulfanilamide \\
\hline Ethosuximide & Theophylline \\
\hline Irinotecan & Tolbutamide \\
\hline Lithium & Drug Abuse/Recreational Drugs \\
\hline Methadone & Amphetamines \\
\hline Metoprolol & Benzodiazepines \\
\hline Oxprenolol & Barbiturates \\
\hline Ethanol & Cocaine \\
\hline Marijuana & Nicotine \\
\hline Opioids & Phencyclidine \\
\hline
\end{tabular}

Table 1. Drug monitoring in saliva
Of particular interest is the use of saliva for the evaluation of illicit drug use. Following drug use, the appearance of the drug in saliva follows a time course that is similar to that of serum. The presence of illicit drugs, and not their concentration, is usually sufficient for forensic purposes.

Ethanol is unionized in serum, is not protein-bound, and has a low molecular weight and lipid solubility. As a result it diffuses rapidly into saliva. Consequently, the saliva-to-serum ratio is generally about 1 . Salivary ethanol concentration may be used as an index of the blood ethanol concentration, provided that the salivary sample is obtained at least $20 \mathrm{~min}$ following ingestion. This will allow for absorption and distribution of alcohol, and prevent a falsely elevated reading due to the oral route of consumption.

Other recreational drugs that can be identified in saliva are amphetamines, barbiturates, benzodiazepines, cocaine, phencyclidine (PCP), and opioids (24).

Nicotine saliva can be used to monitor tobacco smoking and exposure to tobacco smoke. The major nicotine metabolite cotinine was investigated as an indicator of exposure to tobacco smoking. Cotinine is tobacco-specific and has a relatively long half-life (17hours) compared with nicotine. Salivary cotinine levels were found to be indicative of active and passive smoking. Monitoring level of salivary cotinine has proven useful in monitoring compliance with smoking cessation programs (25).

\section{The Monitoring of Hormone Levels}

Saliva can be analyzed as part of the evaluation of endocrine function.

Cortisol Due to their lipid solubility, steroid hormones can be detected in saliva. Salivary cortisol levels were found to be useful in identifying patients with Cushing's syndrome, Addison's disease and the effect of stress (26).

Aldosterone Recent evidence demonstrates an increased incidence of primary aldosteronism (PA) in approximately $10 \%$ of the hypertensive population, making noninvasive and simple screening methods necessary. Salivary aldosterone correlated significantly to plasma aldosterone levels $(r=0.60)$, and increased aldosterone levels were found in both the serum and saliva of patients with primary aldosteronism (Conn's syndrome) (27).

Testosterone and dehydroepiandrosterone have also been identified in saliva. Salivary concentrations were found to be $1.5-7.5 \%$ of the serum concentrations of these hormones. Correlation between the salivary testosterone concentration and the serum free testosterone concentration is better than with total serum testosterone. Because most of the testosterone in the saliva is in the free form (28), Monitoring salivary testosterone levels may be useful to assess testicular function and behavioral studies of aggression, depression, abuse, and violent and antisocial behavior. 
Progesterone - Salivary progesterone levels showed good correlation with free serum levels. Elevated salivary estriol is associated with increased risk of preterm birth (29).

Insulin - Insulin can be detected in saliva, a positive correlation between saliva and serum insulin levels following a glucose tolerance test was reported for healthy subjects, diabetic patients. But the use of salivary insulin levels for the evaluation of serum insulin levels could be misleading, since significant discrepancies between salivary and serum insulin levels were detected for several individuals (30). Additional studies are required to determine if salivary insulin levels should be used for the evaluation of serum insulin levels. Saliva also contains multiple components whose concentrations are altered by diabetes, some of which (glucose, $\alpha$-amylase, and ghrelin) have strong diagnostic potential (31).

In general, serum and salivary levels of protein hormones are not well-correlated. These hormones are too large to reach saliva by means of passive diffusion across cells or by ultrafiltration, and the detection of these hormones in saliva is primarily due to contamination from serum through GCF or oral wounds. Protein hormones such as gonadotrophins, prolactin, and thyrotropin cannot be accurately monitored by means of salivary analysis (32).

7. Bone Turnover Marker in Saliva

Saliva can be used to measure bone turnover. Mcgehee and johnson used commercially available ELISA to test for the presence of osteocalcin (OC) and pyridinoline (PYD) in the whole human saliva of women. Level of OC and PYD in saliva correlated reasonably well with calcaneus bone mineral density BMD/t scores. Suggesting that saliva may be valuable tool for assessing human markers of bone turnover. Further research is necessary to determine whether salivary level of bone turnover marker correlate with serum (33).

\section{Forensic Evidence}

Saliva may be found on victims of several violent crimes. Saliva can potentially be recovered from bite marks, cigarette butts, postage stamps, envelopes and other objects. Stains of dried saliva are invisible, making its recognition and collection difficult. However, the presence of saliva can be confirmed by amylase assay. During the biting process, saliva is deposited on the skin or object surface in enough amount to allow typing of the deoxyribonucleic acid (DNA). Polymerase chain reaction (PCR) allows replication of thousands of copies of a specific DNA sequence in vitro, enabling the study of small amounts of DNA (34).

9. Dental Caries and Periodontal Disease

Dental Caries Counts of mutans streptococci and lactobacilli, Saliva secretion rate and buffering capacity have proven to be sensitive parameters in caries prediction models.

High numbers of S.Mutans and Lactobacillus indicate a shift in oral microflora from healthy to more cariogenic. Diagnostic kits for S. mutans and Lactobacillus counting are widely used in dental practice and can be conducted without laboratory facilities.

In a healthy situation, there is no correlation between saliva secretion rate and dental caries (35). However, when the salivary secretion rate drops below a certain minimum, the amount of dental caries increases dramatically, salivary secretion rate is easily measured by weighing the saliva volume that is collected by expectoration divided by the collection time. Low salivary buffering capacity is a risk factor for dental caries and also is indicative for low saliva secretion. Commercial kits are available for determination of the salivary buffering capacity. Numerous studies have aimed at finding a correlation between dental caries and saliva constituents with only weak correlations. High bacterial aggregation activity of saliva has been associated with low caries experience (36). Since there is a long list of salivary proteins that bind and aggregate oral bacteria so aggregation couldn't be correlated with a specific salivary protein.

Periodontal Disease Another oral disease, for which salivary diagnostics are evaluated, is periodontal disease. Progress from gingivitis to periodontal disease is determined by genetic susceptibility, and the presence of pathogenic bacteria.

There is a large, genetically determined, variation in susceptibility for periodontal disease (37). Mutations in the cathepsin $\mathrm{C}$ gene have been identified as causal for the Papillon-Lefèvre syndrome. In addition, multiple genes have been associated with less severe forms of periodontal disease. People at high risk for periodontal disease can be determined by genetic screening. DNA can easily be isolated from oral epithelial cells, collected by use of a buccal swab, one of the most common oral diagnostics. The loss of attachment and deepening of the periodontal pocket leads to increased leakage of a serum-like fluid designated gingival crevicular fluid, into the oral cavity. Since serum has a 50 to 70 fold higher protein concentration the average protein concentrations in saliva increases dramatically and the concentration of albumin shows an 8-fold increase. During active periods of the disease increased levels of inflammatory markers, like interleukins, can be demonstrated in saliva.

Several bacteria have been associated with periodontal disease these bacteria are susceptible to different antibiotics. Therefore, prior to antibiotic treatment pathogens should be determined by culturing or PCR techniques. Oral fluid may be used for that, or small methylcellulose paper strips can be used to collect fluid from the gingival crevice.

Nevertheless, the recent focuses on the potential role of periodontal disease as a risk factor for cardiovascular and cerebrovascular diseases bring new importance to this aspect of salivary analysis (38). 


\begin{tabular}{|l|}
\hline SYSTEMIC DISEASES AFFECTING SALIVARY \\
GLANDS AND SALIVA \\
\hline $\begin{array}{l}\text { Sjögren's syndrome, rheumatoid diseases, } \\
\text { myasthenia gravis }\end{array}$ \\
\hline Cancer \\
\hline Cirrhosis \\
\hline Cystic fibrosis \\
\hline HIV infection \\
\hline Hypertension \\
\hline Hormonal disorders \\
\hline Malnutrition, dehydration, vitamin deficiency \\
\hline Parkinsonism, Alzheimer's disease \\
\hline Renal disease \\
\hline Sarcoidosis \\
\hline
\end{tabular}

Table 2. Systemic disease affecting salivary gland and saliva

\section{Diagnosis of Oral Disease with Relevance for Sys-} temic Diseases

Some systemic diseases affect salivary glands directly or indirectly, and may influence the quantity and quality of saliva. These characteristic changes may contribute to the diagnosis and early detection of these diseases. Systemic disorders that may affect salivary glands and saliva are presented in Table 2 .

Evaluation of whole saliva quantity may provide information which has systemic relevance. Quantitative alterations in saliva may be a result of medications. At least 400 drugs may induce xerostomia (39). (Diuretics, antihypertensives, antipsychotics, antihistamines, antidepressants, anticholinergics, antineoplastics, and recreational drugs such as opiates, amphetamines, barbiturates, hallucinogens, cannabis, and alcohol ). Reduced salivary flows may lead to progressive dental caries, fungal infection, oral pain, and dysphagia. The reasons for such clinical findings should be thoroughly investigated, since they may be signs of an underlying systemic problem.

Qualitative changes in salivary composition can also provide diagnostic information concerning oral problems. Increased levels of albumin in whole saliva were detected in patients who received chemotherapy and subsequently developed stomatitis. Monitoring of salivary albumin can assist in the identification of stomatitis at a pre-clinical stage and enable the treatment for the stomatitis to be initiated at an early stage. Similarly epidermal growth factor (EGF) in saliva is decreased during the course of radiotherapy and severity of mucositis is related to reduced EGF concentration in saliva (40).

Salivary proteomes will be comprehensively deciphered, catalogued, annotated, and available to the scientific community. Great progress is already on the way with the identification of salivary proteins. The entire content of the salivary proteome in healthy subjects can be viewed at the UCLA data centralization site (www. hspp.ucla.edu). It is envisioned that with the human salivary proteome available, one can begin to exam and compare salivary proteomes of high impact disease such as sjogren syndrome, osteoporosis, rheumatoid arthritis, diabetes, and cancers.

\section{Conclusion}

Although blood is still the gold standard for diagnostics of diseases and drugs, Saliva offers an alternative to serum as a biologic fluid for diagnostic purposes. Saliva is particularly useful for the detection of viral infection (especially HIV due to the non-invasive method of collection), the detection of illicit drug use, monitoring of hormone levels, especially steroids. The functional value of saliva has long been thought to outweigh its diagnostic possibilities. With current research gap is closing rapidly between the use of saliva and other biofluids for disease diagnostics. The emerging field of Microbiology and nanotechnology based biosensor will overcome the detection barriers. It is almost certain that next few years will witness an evolving spectrum of salivary screening. Even home testing kits will begin to appear and saliva will outperform other biomedia in the diagnosis of diseases.

\section{References}

1. Slomiany BL, Aono M, Murty VL, Slomiany A, Levine MJ, Tabak LA. Lipid composition of submandibular saliva from normal and cystic fibrosis individuals. J Dent Res. 1982;61:1163-6.

2. Ueshiba H, Zerah M, new MI. Enzyme-linked immunosorbent assay (ELISA) method for screening of non-classical steroids 21hydroxylase deficiency. Horm Metab Res. 1994;26:43-5.

3. Iwasaki K, Okawa-Takatsuji M, Aotsuka S, Ono T. Detection of anti-SS-A/Ro and anti-SS-B/La antibodies of IgA and IgG isotypes in saliva and sera of patients with Sjogren's syndrome. Nihon Rinsho Meneki Gakkai Kaishi. 2003;26:346-54.

4. Warnakulasuriya S, Soussi T, Maher R, Johnson N, Tavassoli M. Expression of p53 in oral squamous cell carcinoma is associated with the presence of IgG and IgA p53 autoantibodies in sera and saliva of the patients. J Pathol. 2000;192:52-7.

5. Mizukawa N, Sugiyama K, Fukunaga J, Ueno T, Mishima K, Takagi $\mathrm{S}$, et al. Defensin-1, a peptide detected in the saliva of oral squamous cell carcinoma patients. Anticancer Res. 1998;18:4645-9.

6. Agha-Hosseini F, Mirzaii-Dizgah I, Rahimi A. Correlation of serum and salivary CA15-3 levels in patients with breast cancer. Med Oral Patol Oral Cir Bucal. 2009;14:e521-4.

7. Agha-Hosseini F, Mirzaii-Dizgah I, Rahimi A, Seilanian-Toosi M. Correlation of serum and salivary CA125 levels in patients with breast cancer. J Contemp Dent Pract. 2009;10:E001-8.

8. Badawi AF, Hosny G, El-Hadary M, Mostafa MH. Salivary nitrate, nitrite and nitrate reductase activity in relation to risk of oral cancer in Egypt. Dis Markers. 1998;14:91-7.

9. Kabir S. Detection of helicobacter pylori DNA in feces and saliva by polymerase chain reaction: a review. Helicobacter. 2004;9:11523.

10. Schultsz C, Qadri F, Hossain SA, Ahmed F, Ciznar I. Shigellaspecific IgA in saliva of children with bacillary dysentery. FEMS Microbiol Immunol. 1992;4:65-72.

11. Foo RL, Graham SM, Suthisarnsuntorn U, Parry CM. Detection of pneumococcal capsular antigen in saliva of children with pneumonia. Ann Trop Paediatr. 2000;20:161-3.

12. Schwartz BS, Ford DP, Childs JE, Rothman N, Thomas RJ. Anti-tick saliva antibody: a biologic marker of tick exposure that is a risk factor for Lyme disease seropositivity. Am J Epidemiol. 1991;134:86-95. 
13. Bueno EC, Vaz AJ, Machado LD, Livramento JA. Neurocysticercosis: detection of $\operatorname{IgG}, \operatorname{IgA}$ and $\operatorname{IgE}$ antibodies in cerebrospinal fluid, serum and saliva samples by ELISA with Taenia solium and Taenia crassiceps antigens. Arq Neuropsiquiatr. 2000;58:18-24.

14. Haque R, Kabir M, Noor Z, Rahman SM, Mondal D, Alam F, et al. Diagnosis of amebic liver abscess and amebic colitis by detection of Entamoeba histolytica DNA in blood, urine, and saliva by a realtime PCR assay. J Clin Microbiol. 2010;48:2798-801.

15. Malamud D. Oral diagnostic testing for detecting human immunodeficiency virus-1 antibodies: a technology whose time has come. Am J Med. 1997;102:9-14.

16. Cordeiro ML, Turpin CS, McAdams SA. A comparative study of saliva and OraSure oral fluid. Ann NY Acad Sci.1993;694:330-1.

17. Oba IT, Spina AM, Saraceni CP, Lemos MF, Senhoras R, Moreira $\mathrm{RC}$, et al. detection of hepatitis A antibodies by ELISA using saliva as clinical samples. Rev Inst Med Trop Sao Paulo. 2000;42:197200 .

18. Zhang YL, Pan HY, Chen CR, Lou GQ, YE RX, Lu DR. The roles of saliva testing for preventing hepatitis $\mathrm{B}$ virus spreading. Zhonghua Yu Fang Yi Xue Za Zhi. 2008;42:596-8.

19. Jin L, Vyse A, Brown DW. The role of RT-PCR assay of oral fluid for diagnosis and surveillance of measles, mumps and rubella. Bull World Health Organ. 2002;80:76-7.

20. Aiyar J, Bhan MK, Bhandari N, Kumar R, Raj P, Sazawal S. Rotavirus-specific antibody response in saliva of infants with rotavirus diarrhea. J Infect Dis. 1990;162:1383-4.

21. Lazarini PR, Vianna MF, Alcantara MP, Scalia RA, Caiaffa Filho $\mathrm{HH}$. Herpes simplex virus in the saliva of peripheral Bell's palsy patients. Braz J Otorhinolaryngol. 2006;72:7-11.

22. Chakravarti A, Matlani M, Jain M. Immunodiagnosis of dengue virus infection using saliva. Curr Microbiol. 2007;55:461-4.

23. Vasudev A, Tripathi KD, Puri V. Correlation of serum and salivary carbamazepine concentration in epileptic patients: implications for therapeutic drug monitoring. Neurol India. 2002;50:60-2.

24. Cone EJ, Huestis MA . Interpretation of oral fluid tests for drugs of abuse. Saliva testing for drugs of abuse. Ann NY Acad Sci. 2007;1098:51-103.

25. Figueiredo VC, Szklo M, Szklo AS, Benowitz N, Lozana JA, Casado L, et al. Determinants of salivary cotinine level: a populationbased study in Brazil. Rev Saude Publica. 2007;41:954-62.

26. Raff H. Utility of salivary cortisol measurements in Cushing's syndrome and adrenal insufficiency. J Clin Endocrinol Metab. 2009;94:3647-55.

27. Manolopoulou J, Mulatero P, Maser-Gluth C, Rossignol P, Spyroglou A, Vakrilova Y, et al. Saliva as a medium for aldosterone measurement in repeated sampling studies. Steroids. 2009;74:853-8.

28. Jinrui H, Itoh N, Nitta T, Kurohata T,Tsukamoto T, Kumamoto Y, et al. Changes in the salivary testosterone level in aged. Hinyokika Kiyo. 1994;40:807-11.

29. Heine RP, McGregor JA, Goodwin TM, Artal R, Hayashi RH, Robertson PA, Varner MW. Serial salivary estriol to detect an increased risk of preterm birth. Obstet Gynecol. 2000;96:490-7.

30. Pasic J, Pickup JC. Salivary insulin in normal and type I diabetic subjects. Diabetes Care. 1988;11:489-494.

31. Aydin S. A comparison of ghrelin, glucose, alpha-amylase and protein levels in saliva from diabetics.J Biochem Mol Biol. 2007;40:29-35.

32. Groschl M. Current status of salivary hormone analysis. Ann Biol Clin (Paris). 2009;67:493-504.

33. McGehee JW Jr, Johnson RB. Biomarkers of bone turnover can be assayed from human saliva. J Gerontol A Biol Sci Med Sci. 2004;59:196-200.

34. Sweet D, Hildebrand D. Saliva from cheese bite yields DNA profile of burglar: a case report. Int J Legal Med. 1999;112:201-3.

35. Lenander-Lumikari M, Loimaranta V. Saliva and dental caries. Adv Dent Res. 2000;14:40-7.

36. Van Nieuw Amerongen A, Bolscher JG, Veerman EC. Salivary proteins: protective and diagnostic value in cariology? Caries Res. 2004;38:247-53.
37. Pihlstrom BL, Michalowicz BS, Johnson NW. Periodontal disease. Lancet. 2005;366:1809-20.

38. Morrison HI, Ellison LF, Taylor GW. Periodontal disease and risk of fatal coronary heart and cerebrovascular disease. J Cardiovasc Risk. 1999;6:7-11.

39. Rees TD. Drugs and oral disorders. Periodontol 2000. 1998;18:2136.

40. Epstein JB, Gorsky M, Guglietta A, Le N, Sonis ST. The correlation between epidermal growth factor levels in saliva and the severity of oral mucositis during oropharyngeal radiation therapy. Cancer. 2000;89:2258-65. 